\title{
COMPACT COMPOSITION OPERATORS ON BLOCH SPACES
}

\author{
Chengu Xiong
}

The present paper proposes new criteria for compactness of a composition operator $C_{\phi} f=f \circ \phi$ on the Bloch space and the little Bloch space. For the case when $\phi$ is univalent, a criterion given by $\mathrm{K}$. Madigan and A. Matheson is generalised.

\section{INTRODUCTION}

Let $D$ denote the unit disk in the complex plane. A function $f$ holomorphic on $D$ is said to belong to the Bloch space $\mathcal{B}$ if

$$
\sup _{z \in D}\left(1-|z|^{2}\right)\left|f^{\prime}(z)\right|<\infty
$$

and to the little Bloch space $\mathcal{B}_{0}$ if

$$
\lim _{|z| \rightarrow 1^{-}}\left(1-|z|^{2}\right)\left|f^{\prime}(z)\right|=0 .
$$

It is well known that $\mathcal{B}$ is a Banach space under the norm

$$
\|f\|_{\mathcal{B}}=|f(0)|+\sup _{z \in D}\left(1-|z|^{2}\right)\left|f^{\prime}(z)\right|
$$

and that $\mathcal{B}_{0}$ is a closed subspace of $\mathcal{B}$. A good source for results and references concerning Bloch functions is the paper of Anderson, Clunie and Pommerenke ([1]).

It is known that a holomorphic mapping $\phi$ of $D$ into itself induces a bounded composition operator $C_{\phi} f=f \circ \phi$ on $\mathcal{B}$, and $C_{\phi}$ is a composition operator on $\mathcal{B}_{0}$ if and only if $\phi \in \mathcal{B}_{0}([2])$. Madigan and Matheson ([2]) studied the compactness of composition operators and obtained the following results.

THEOREM A. If $\phi$ is a holomorphic mapping of $D$ into itself, then $C_{\phi}$ is a compact operator on $\mathcal{B}_{0}$ if and only if

$$
\lim _{z \rightarrow D D} \frac{1-|z|^{2}}{1-|\phi(z)|^{2}}\left|\phi^{\prime}(z)\right|=0
$$

Received 19th November, 2002

Copyright Clearance Centre, Inc. Serial-fee code: 0004-9727/03 \$A2.00+0.00. 
TheOREM B. If $\phi$ is a holomorphic mapping of $D$ into itself, then $C_{\phi}$ is a compact operator on $\mathcal{B}$ if and only if for any $\varepsilon>0$ there exists $r, 0<r<1$, such that

$$
\sup _{|\phi(z)|>r} \frac{1-|z|^{2}}{1-|\phi(z)|^{2}}\left|\phi^{\prime}(z)\right|<\varepsilon .
$$

Theorem $\mathrm{C}$. If $\phi$ is univalent and if $G=\phi(D)$ has a nontangential cusp at 1 and touches $\partial D$ at no other point, then $C_{\phi}$ is a compact operator on $\mathcal{B}_{0}$.

In the same paper, Madigan and Matheson proposed two questions:

1. If $\phi \in \mathcal{B}_{0}$ and $C_{\phi}$ is compact, then $\log (1 / 1-\bar{w} \phi(z)) \in \mathcal{B}_{0}$ for all $w \in \partial D$. Is the converse of this true?

2. Is there a $\phi \in \mathcal{B}_{0}$ such that $\overline{\phi(D)} \cap \partial D$ is infinite and $C_{\phi}$ is compact on $\mathcal{B}_{0}$ ?

In Section 2 of this paper, we discuss Question 1 and give an example which yields a negative anwser. At the same time, we show that $C_{\phi}$ is compact on $\mathcal{B}_{0}$ if and only if $\log (1 / 1-\bar{w} \phi(z)) \in \mathcal{B}_{0}$ uniformly for all $w \in \partial D$. Section 3 is devoted to the situation when $\phi$ is a univalent holomorphic self-mapping of $D$. Theorem $C$ is generalised to the case that $\overline{\phi(D)} \cap \partial D$ contains countably many points, and an example which satisfies the conditions in Question 2 is constructed.

\section{QUESTION ONE}

We shall construct a function $\phi$ which furnishes a negative answer for Question 1 . Let

$$
G=\left\{r e^{i \theta}: 0<\theta<\frac{\pi}{16}, 1-3 \theta^{2}<r<1-\theta^{2}\right\} .
$$

By the Riemann mapping theorem, there exists a conformal mapping $\phi$ of $D$ onto $G$. It is known that $\phi$ belongs to $\mathcal{B}_{0}([4])$ and $C_{\phi}$ is a bounded composition operator on $\mathcal{B}_{0}$.

First, we show that $C_{\phi}$ is not compact on $\mathcal{B}_{0}$. For $0<\theta<\pi / 16$, let $z(\theta) \in D$ be such that $\phi(z(\theta))=w(\theta)=\left(1-2 \theta^{2}\right) e^{i \theta} \in G$. Then

$$
\begin{aligned}
\operatorname{dist}(w(\theta), \partial G) & =\inf _{0<\alpha<\pi / 16}\left\{\left|\left(1-2 \theta^{2}\right) e^{i \theta}-\left(1-\alpha^{2}\right) e^{i \alpha}\right|,\left|\left(1-2 \theta^{2}\right) e^{i \theta}-\left(1-3 \alpha^{2}\right) e^{i \alpha}\right|\right\} \\
& \geqslant \theta^{2}
\end{aligned}
$$

Using the Koebe distortion theorem [3] for $\phi$, we have

$$
\left(1-|z(\theta)|^{2}\right)\left|\phi^{\prime}(z(\theta))\right| \geqslant \operatorname{dist}(\phi(z(\theta)), \partial G) \geqslant \theta^{2} \text {. }
$$

So,

$$
\frac{1-|z(\theta)|^{2}}{1-|w(\theta)|^{2}}\left|\phi^{\prime}(z(\theta))\right| \geqslant \frac{\theta^{2}}{1-\left(1-2 \theta^{2}\right)^{2}} \geqslant \frac{1}{4} \text { for } 0<\theta<\frac{\pi}{16} .
$$

Note that $|z(\theta)| \rightarrow 1$ as $\theta \rightarrow 0$. By theorem $\mathrm{A}, C_{\phi}$ is not compact. 
Now, let $w \in \partial D$ be fixed and $f(z)=\log (1 / 1-\bar{w} \phi(z))$. Then

$$
\left(1-|z|^{2}\right)\left|f^{\prime}(z)\right|=\frac{1-|z|^{2}}{|1-\bar{w} \phi(z)|}\left|\phi^{\prime}(z)\right| .
$$

If $w \neq 1$, there exists a positive number $\delta$ such that $|1-\bar{w} \phi(z)|>\delta>0$ for $z \in D$. Thus $f \in \mathcal{B}_{0}$ since $\phi \in \mathcal{B}_{0}$. If $w=1$, assume for the sake of contradiction that $f(z) \notin \mathcal{B}_{0}$. By Theorem $A$, there exists a sequence $\left\{z_{n}\right\}$ and a positive number $\varepsilon$, such that $\left|z_{n}\right| \rightarrow 1$ and

$$
\left(1-\left|z_{n}\right|^{2}\right)\left|f^{\prime}\left(z_{n}\right)\right|=\frac{1-\left|z_{n}\right|^{2}}{\left|1-\phi\left(z_{n}\right)\right|}\left|\phi^{\prime}\left(z_{n}\right)\right|>\varepsilon .
$$

Without loss of generality, we may assume $w_{n}=\phi\left(z_{n}\right) \rightarrow w_{0}$. If $w_{0} \neq 1$, the above inequality contradicts the fact $\phi(z) \in \mathcal{B}_{0}$. If $w_{0}=1$, using the Schwarz-Pick lemma, we have

$$
\begin{aligned}
\left(1-\left|z_{n}\right|^{2}\right)\left|f^{\prime}\left(z_{n}\right)\right| & =\frac{1-\left|z_{n}\right|^{2}}{1-\left|\phi\left(z_{n}\right)\right|^{2}}\left|\phi^{\prime}\left(z_{n}\right)\right| \cdot \frac{1-\left|\phi\left(z_{n}\right)\right|^{2}}{\left|1-\phi\left(z_{n}\right)\right|} \\
& \leqslant\left(1+\left|w_{n}\right|\right) \frac{1-\left|w_{n}\right|}{\left|1-w_{n}\right|} \rightarrow 0,
\end{aligned}
$$

which leads to a contradiction. This shows that $\log (1 / 1-\bar{w} \phi(z)) \in \mathcal{B}_{0}$ for all $w \in \partial D$.

The above example shows that the condition $f(z, w)=\log (1 / 1-\bar{w} \phi(z)) \in \mathcal{B}_{0}$ for all $w \in \partial D$ does not guarantee the compactness of $C_{\phi}$. However, if we assume that $f(z, w)$ belongs to $\mathcal{B}_{0}$ uniformly for $w \in \partial D$, that is, for any $\varepsilon>0$, there exists $r, 0<r<1$, such that $\left(1-|z|^{2}\right)\left|f_{z}^{\prime}(z, w)\right|<\varepsilon$ for $r<|z|<1$ and $w \in \partial D$, then $C_{\phi}$ will be compact.

THEOREM 1. If $\phi \in \mathcal{B}_{0}$, then the composition operator $C_{\phi}$ on $\mathcal{B}_{0}$ is compact if and only if $f(z, w) \in \mathcal{B}_{0}$ uniformly for $w \in \partial D$.

Proof: First, assume that $\phi \in \mathcal{B}_{0}$ and $C_{\phi}$ is compact on $\mathcal{B}_{0}$. A direct computation gives

$$
\begin{aligned}
\left(1-|z|^{2}\right)\left|f_{z}^{\prime}(z, w)\right| & =\left(1-|z|^{2}\right)\left|\frac{\phi^{\prime}(z)}{1-\bar{w} \phi(z)}\right| \\
& =\frac{\left(1-|z|^{2}\right)\left|\phi^{\prime}(z)\right|}{1-|\phi(z)|^{2}} \cdot \frac{1-|\phi(z)|^{2}}{|1-\bar{w} \phi(z)|} \\
& \leqslant \frac{2\left(1-|z|^{2}\right)\left|\phi^{\prime}(z)\right|}{1-|\phi(z)|^{2}} .
\end{aligned}
$$

Since $C_{\phi}$ is compact on $\mathcal{B}_{0}$, using theorem $\mathrm{A}$, we have

$$
\lim _{z \rightarrow \partial D} \frac{1-|z|^{2}}{1-|\phi(z)|^{2}}\left|\phi^{\prime}(z)\right|=0 .
$$

Thus, $f(z, w)$ belongs to $\mathcal{B}_{0}$ uniformly for $w \in \partial D$. The necessity is proved. 
Now, let $f(z, w)$ belong to $\mathcal{B}_{0}$ uniformly for $w \in \partial D$. Then, for every $\varepsilon>0$, there exists $r, 0<r<1$, such that

$$
\begin{aligned}
\left(1-|z|^{2}\right)\left|f_{z}^{\prime}(z, w)\right| & =\frac{\left(1-|z|^{2}\right)\left|\phi^{\prime}(z)\right|}{1-|\phi(z)|^{2}} \cdot \frac{1-|\phi(z)|^{2}}{|1-\bar{w} \phi(z)|} \\
& <\varepsilon \text { for }|z|>r, w \in \partial D .
\end{aligned}
$$

For $|z|>r$, letting $w=\phi(z) /|\phi(z)|$ in the above inequality, we have

$$
\frac{\left(1-|z|^{2}\right)\left|\phi^{\prime}(z)\right|}{1-|\phi(z)|^{2}}<\varepsilon .
$$

By Theorem $\mathrm{A}, C_{\phi}$ is compact on $\mathcal{B}_{0}$. The theorem is proved.

In the same way as above, we can prove the following theorem for composition operators on $\mathcal{B}$.

THEOREM 2. For any holomorphic mapping $\phi$ of $D$ into itself, the composition operator $C_{\phi}$ is compact on $\mathcal{B}$ if and only if for any $\varepsilon>0$ there exists $r, 0<r<1$, such that

$$
\sup _{|\phi(z)|>r}\left(1-|z|^{2}\right)\left|f_{z}^{\prime}(z, w)\right|<\varepsilon \quad \text { for } r<|z|<1, w \in \partial D
$$

\section{UNIVALENT CASE}

In this section, we consider only the case that $\phi(z)$ is a univalent mapping of $D$ into itself. It is well known that such a $\phi$ belongs to the little Bloch space $\mathcal{B}_{0}([4])$, and $C_{\phi}$ is a bounded composition operator on $\mathcal{B}_{0}$. Denote $G=\phi(D) \subset D$. For $w_{0} \in \bar{G} \cap \partial D$, we say $G$ has a cusp at $w_{0}$ if there exists a neighbourhood $U$ of $w_{0}$ such that $U \cap \bar{G} \cap \partial D=\left\{w_{0}\right\}$, and

$$
\operatorname{dist}(w, \partial G)=o\left(\left|w_{0}-w\right|\right)
$$

as $w \rightarrow w_{0}$ in $G$. The cusp $w_{0}$ is said to be nontangential if $U \cap G$ lies inside a Stolz angle near $w_{0}$, that is, there exist $r, M>0$ such that

$$
\left|w_{0}-w\right| \leqslant M\left(1-|w|^{2}\right) \quad \text { for }\left|w_{0}-w\right|<r, w \in G \cap U
$$

In Theorem $\mathrm{C}$, it is assumed that $\bar{G}$ intersects $\partial D$ at one point 1 . It is obvious that Theorem $C$ is valid when $G$ touches the unit circle at only a finite number of points all of which are nontangential cusps. Now, we generalise this to the case that $\bar{G} \cap \partial D$ contains infinitely many points.

TheOREM 3. Let $\phi$ be a univalent holomorphic mapping of $D$ into itself and $G=\phi(D)$. If $\operatorname{dist}(w, \partial G)=o(1-|w|)$ as $w \rightarrow \partial D$, then $C_{\phi}$ is compact on $\mathcal{B}_{0}$. 
Proof: By the hypothesis of the theorem, for $\varepsilon>0$, there exists $r, 0<r<1$, such that

$$
\operatorname{dist}(w, \partial G)<\varepsilon(1-|w|) \text { for }|w|>r .
$$

Since $\phi \in \mathcal{B}_{0}$, there exists $r^{\prime}, 0<r^{\prime}<1$, such that

$$
\left(1-|z|^{2}\right)\left|\phi^{\prime}(z)\right|<\left(1-r^{2}\right) \varepsilon \text { for }|z|>r^{\prime} .
$$

Let $z$ be a point such that $|z|>r^{\prime}$ and $w=\phi(z)$. If $|w|>r$, let $\psi$ be a holomorphic mapping of $D$ onto itself such that $\psi(0)=z$. Then, by Koebe's one-quarter theorem,

$$
\left(1-|z|^{2}\right)\left|\phi^{\prime}(z)\right|=(\phi \circ \psi)^{\prime}(0) \leqslant 4 \operatorname{dist}(w, \partial G) .
$$

Thus,

$$
\frac{\left(1-|z|^{2}\right)\left|\phi^{\prime}(z)\right|}{1-|\phi(z)|^{2}}<\frac{4 \varepsilon}{1+|\phi(z)|}<4 \varepsilon
$$

If $|\phi(z)| \leqslant r$, we have

$$
\frac{\left(1-|z|^{2}\right)\left|\phi^{\prime}(z)\right|}{1-|\phi(z)|^{2}}<\frac{\left(1-r^{2}\right) \varepsilon}{1-|\phi(z)|^{2}} \leqslant \varepsilon .
$$

By Theorem $\mathrm{A}, C_{\phi}$ is compact. The theorem is proved.

COROLLARY 1. Let $\phi$ be a univalent holomorphic mapping of $D$ into itself and $G=\phi(D)$. For $0<r<1$, denote the maximum length of all arcs contained in $G \cap\{z$ : $|z|=r\}$ by $l(r)$. If $l(r) /(1-r) \rightarrow 0$ as $r \rightarrow 1$, then $C_{\phi}$ is compact on $\mathcal{B}_{0}$.

We now present an example of a $\phi$ for which $G=\phi(D)$ satisfies the condition in the above corollary and $\bar{G} \cap \partial D$ contains infinitely many points. This gives a positive answer to Question 2. Let

$$
\begin{aligned}
D_{0} & =\left\{z:|z|<\frac{1}{2}\right\} \\
A\left(\delta, \theta_{0}\right) & =\left\{r e^{i \theta}: \theta_{0}-\delta<\theta<\theta_{0}+\delta, \frac{1}{2} \leqslant r<1-\frac{1}{2 \sqrt{\delta}} \sqrt{\left|\theta-\theta_{0}\right|}\right\} .
\end{aligned}
$$

Denote

$$
\begin{aligned}
G & =D_{0} \cup A\left(\frac{\pi}{8}, \frac{\pi}{8}\right) \cup A\left(\frac{\pi}{16}, \frac{5 \pi}{16}\right) \cup A\left(\frac{\pi}{32}, \frac{13 \pi}{32}\right) \cup \cdots \\
& =D_{0} \bigcup_{i=1}^{\infty} A\left(\frac{1}{2^{i+2}} \pi, \frac{2^{i+1}-3}{2^{i+2}} \pi\right) .
\end{aligned}
$$

It is easy to verify that $G \subset D$ is a simply connected domain and satisfies the condition in Corollary 1. Let $\phi$ be a conformal mapping of $D$ onto $G$. Then, by Corollary $1, C_{\phi}$ is compact on $\mathcal{B}_{0}$. 


\section{REFERENCES}

[1] J.M. Anderson, J. Clunie and Ch. Pomerenke, 'On Bloch functions and normal functions', J. Reine Angew.Math. 270 (1974), 12-37.

[2] K. Madigan and A. Matheson, 'Compact composition operators on Bloch space', Trans. Amer. Math. Soc. 347 (1995), 2679-2687.

[3] Ch. Pommerenke, Univalent functions (Vandenhoeck and Ruprecht, Göttingen, 1975).

[4] Ch. Pommerenke, Boundary behavior of conformal maps (Springer-Verlag, Berlin, 1992).

Department of Mathematics

Nanjing Normal University

Nanjing 210097

China
Department of Mathematics

Huaiyin Teachers' College

Huiayin 223001

China

e-mail: chengii_xiong@163.net 